\title{
Ursodeoxycholic Acid (UDCA) Exerts Anti- Atherogenic Effects by Inhibiting Endoplasmic Reticulum (ER) Stress Induced by Disturbed Flow
}

\author{
Jihwa Chung ${ }^{1,3}$, Kyoung Hwa Kim ${ }^{1,3}$, Seok Cheol Lee ${ }^{1}$, Shung Hyun An ${ }^{1}$, and Kihwan Kwon ${ }^{1,2, *}$
}

\begin{abstract}
Disturbed blood flow with low-oscillatory shear stress (OSS) is a predominant atherogenic factor leading to dysfunctional endothelial cells (ECs). Recently, it was found that disturbed flow can directly induce endoplasmic reticulum (ER) stress in ECs, thereby playing a critical role in the development and progression of atherosclerosis. Ursodeoxycholic acid (UDCA), a naturally occurring bile acid, has long been used to treat chronic cholestatic liver disease and is known to alleviate endoplasmic reticulum (ER) stress at the cellular level. However, its role in atherosclerosis remains unexplored. In this study, we demonstrated the anti-atherogenic activity of UDCA via inhibition of disturbed flow-induced ER stress in atherosclerosis. UDCA effectively reduced $E R$ stress, resulting in a reduction in expression of X-box binding protein-1 (XBP-1) and CEBPhomologous protein (CHOP) in ECs. UDCA also inhibits the disturbed flow-induced inflammatory responses such as increases in adhesion molecules, monocyte adhesion to ECs, and apoptosis of ECs. In a mouse model of disturbed flow-induced atherosclerosis, UDCA inhibits atheromatous plaque formation through the alleviation of ER stress and a decrease in adhesion molecules. Taken together, our results revealed that UDCA exerts antiatherogenic activity in disturbed flow-induced atherosclerosis by inhibiting $E R$ stress and the inflammatory response. This study suggests that UDCA may be a therapeutic agent for prevention or treatment of atherosclerosis.
\end{abstract}

\section{INTRODUCTION}

Atherosclerosis is an inflammatory vascular disease that develops preferentially in areas of disturbed flow. Endothelial cells

\footnotetext{
${ }^{1}$ Medical Research Institute, School of Medicine, Ewha Womans University, Seoul 158-710, Korea, ${ }^{2}$ Department of Internal Medicine, Cardiology Division and GT5 Program of Ewha Womans University School of Medicine, Seoul $158-710$, Korea, ${ }^{3}$ These authors contributed equally to this work.

*Correspondence: kankadin@ewha.ac.kr
}

Received 3 April, 2015; revised 8 July, 2015; accepted 17 July, 2015; published online 7 October, 2015

Keywords: atherosclerosis, disturbed flow, endoplasmic reticulum stress, endothelial cells, ursodeoxycholic acid
(ECs) that exist in the innermost layers of blood vessels are exposed to fluid shear stress that modulates endothelial function and vascular pathophysiology (Cunningham and Gotlieb, 2005). Many previous studies have shown that the expression levels of anti-atherogenic genes are upregulated in laminar shear stress (LSS) with high and undirected flow, whereas disturbed flow with low or irregular flow upregulates expression of atherogenic genes (Dai et al., 2004; Heo et al., 2014 Passerini et al., 2004). Therefore, disturbed flow is a main cause of atherosclerosis.

The activation of the unfolded protein response (UPR), referred to as ER stress, plays a pivotal role in the development and progression of atherosclerosis (Hotamisligil, 2010; Tabas, 2009). ER stress is an adaptive response to maintain ER homeostasis, but prolonged ER stress triggers apoptosis and ROS generation (Scull and Tabas, 2011; Zhou et al., 2005). In particular, prolonged elevation of CHOP triggers apoptosis through effects on intracellular calcium metabolism and by alterations in Bcl family members (Lin et al., 2007; Timmins et al., 2009; Zinszner et al., 1998) .

Recently, many studies have shown that disturbed flow directly induces ER stress and promotes progression of atherosclerosis. Zeng et al. (2009). reported that disturbed flow triggers ER stress via activation of XBP-1, resulting in the induction of apoptosis in ECs. In in vivo swine models, the endothelium of athero-susceptible regions with disturbed flow showed upregulation of genes associated with ER stress (Civelek et al., 2009).

UDCA, a hydrophilic bile salt, has been widely used for the treatment of certain cholestatic liver diseases such as primary biliary cirrhosis and chronic viral hepatitis (Beuers et al., 1998; Lindor, 2007). UDCA exerts cytoprotective activity including anti-apoptotic and anti-inflammatory effects, but these activities have previously been described only in hepatocytes. Some researchers have reported on the effects of UDCA in cardiovascular diseases. UDCA increases nitric oxide production and inhibits endothelin-1 production in human vascular ECs (Ma et al., 2004). Taurin-conjugated ursodeoxycholic acid (TUDCA) inhibits neointimal hyperplasia by reducing the proliferation of and inducing apoptosis in the vascular smooth muscle cells of rats subjected to carotid artery balloon injury (Kim et al., 2011). Chemical chaperones, including 4-phenylbutyric acid and TUDCA, have been shown to alleviate ER stress. These facts suggest the possibility that UDCA might exert cytoprotective effects in cardiovascular diseases such as atherosclerosis. 
However, the effects and mechanisms of action of UDCA in atherosclerosis are not fully explored.

In this study, we demonstrate that UDCA has anti-atherogenic effects by blocking the ER stress and inflammatory response induced by disturbed flow in ECs. UDCA suppressed the development of atherosclerotic lesions in a mouse model of disturbed flow-induced atherosclerosis, at least partially inhibiting the activation of XBP-1 and CHOP in the ER stress pathway, thereby down-regulating adhesion molecule expression. Therefore, UDCA may be a potential therapeutic agent for prevention or treatment of atherosclerosis.

\section{MATERIALS AND METHODS}

\section{Cell culture and UDCA treatment}

Human umbilical vein endothelial cells (HUVECs) were cultured in Medium 200 with $5 \%(\mathrm{v} / \mathrm{v})$ fetal bovine serum and low-serum growth supplement (LSGS; Cascade Biologics)(Ha et al., 2008). The human leukemic monocyte lymphoma cell line, U937 cells, were purchased from the Korean Cell Line Bank (KCLB) and cultured in RPMI-1640 medium (Gibco) with 10\% (v/v) FBS at $37^{\circ} \mathrm{C}$ under $5 \%(\mathrm{v} / \mathrm{v}) \mathrm{CO}_{2}$. UDCA was kindly provided by Daewoong Pharmaceutical Co. Ltd. UDCA $(100 \mu \mathrm{M})$ was dissolved in DMSO and administered simultaneously with exposure to fluid shear stress.

\section{Fluid shear stress experiments}

Confluent HUVECs cultured in 60-mm dishes were exposed to fluid shear stress as indicated. Cells were exposed to flow in a cone-and-plate viscometer. We used a unidirectional steady flow (shear stress of $15 \mathrm{dyne} / \mathrm{cm}^{2}$ ) for LSS, and a bidirectional disturbed flow (shear stress of $\pm 5 \mathrm{dyne} / \mathrm{cm}^{2}$ ) for OSS, as previously described (Ha et al., 2013).

\section{Animal model of atherosclerosis induced by disturbed} flow

The animal study was performed in accordance with the Guidelines for Animal Experiments of the Animal Experimentation Ethics Committee of Ewha Womans University. We generated a model of atherosclerosis induced by disturbed flow in mouse by partial carotid artery ligation. Male ApoE KO (Central Lab Animal) mice were ligated at 6 weeks of age. In this model, the endothelium of partially ligated LCA was exposed to low-OSS, endothelial dysfunction was induced within 1 week, and rapid atherosclerosis developed within 2 weeks. Partial ligation of LCA was carried out as described in a previous study (Nam et al., 2009). The mice with partially ligated LCA were divided into two groups, a control group fed a high fat diet (HFD) and a UDCA-treated group fed an HFD with 0.5\% UDCA (equal to approximately $400-600 \mathrm{mg} / \mathrm{kg} /$ day $/$ mouse), both for 2 weeks. Carotid arteries were isolated for Oil-red-O and immunohistochemical staining.

\section{Western blotting}

Cells were harvested and lysed with RIPA buffer containing $1 \%$ $(\mathrm{v} / \mathrm{v})$ protease inhibitor- and phosphatase inhibitor-cocktail (GenDEPOT). Briefly, lysates were centrifuged at $13,000 \mathrm{rpm}$ for $30 \mathrm{~min}$ and supernatants were collected. Protein concentrations in cell lysates were measured using a BCA protein assay kit (Thermo Scientific). Equal amounts of protein were subjected to SDS-PAGE gel electrophoresis. Total protein expression was normalized to GAPDH to control for loading. Antibodies were used to detect expression levels of phospho-PERK (Santa Cruz Biotechnology, 1:500), phospho-elF2 $\alpha$ (Santa Cruz
Biotechnology, 1:1000), CHOP (Santa Cruz Biotechnology, 1:500), XBP-1 (Abcam, 1:500), p50 ATF6 (IMGENEX, 1:1000), eNOS (Cell signaling Technology, 1:1000), phospho-eNOS (Ser1177) (Cell signaling Technology, 1:1000), KLF2 (Abcam, 1:1000), VCAM-1 (Abcam, 1:1000), ICAM-1 (Santa Cruz Biotechnology, 1:1000), and GAPDH (Santa Cruz Biotechnology, 1:1000).

Reverse transcription-polymerase chain reaction (RT-PCR) Total cellular RNA was extracted from HUVECs using a Total RNA Isolation Kit (Qiagen) according to the manufacturer's instructions. Complementary DNA (CDNA) was synthesized using M-MLV reverse transcriptase (Promega) and oligo-dT 15 primer (Promega). cDNA was amplified by PCR over 30 cycles. The following oligonucleotide primers were used in this study: Human eNOS: sense $5^{\prime}$-TGATGCATTGGATCTTTGGA-3' and antisense 5'-CCATGTTACTGTGCGTCCAC-3'; Human KLF2: sense $5^{\prime}$-CCTCCCAAACTGTGACTGGT-3' and antisense $5^{\prime}$ ACTCGTCAAGGAGGATCGTG-3'; Human VCAM-1: sense 5'CCGGATTGCTGCTCAGATTGGA-3' and antisense $5^{\prime}$-AGCG TGGAATTGGTCCCCTCA-3'; Human ICAM-1: sense 5'-GG CCTCAGTCAGTGTGA-3' and antisense $5^{\prime}$-AACCCCATTCAG CGTCA-3'; Human GAPDH: sense 5'-GAGTCAACGGATTTG GTCGT-3' and antisense 5'-TTGATTTTGGAGGGATCTCG-3'.

\section{Monocyte adhesion assay}

HUVECs were cultured under LSS or OSS conditions for $24 \mathrm{~h}$ with or without UDCA, after which U937 cells were added to the dishes followed by incubation for $30 \mathrm{~min}$ at $37^{\circ} \mathrm{C}$. The unbound cells were removed by washing three times with serum-free medium. The adherent cells were counted in five randomly selected optical fields in each well. Phase-contrast microphotographs of the cells in the plates were taken using an Olympus CKX41 inverted microscope (Olympus America).

\section{Assessment of apoptosis}

Apoptotic cells were assessed by an Annexin V-FITC apoptosis detection kit (Sigma-Aldrich) according to the manufacturer's instructions. In brief, HUVECs were cultured under LSS or OSS conditions for $48 \mathrm{~h}$ with or without UDCA. Cells of each group were harvested, washed twice with cold $2 \%$ FBS in PBS, resuspended in $500 \mu \mathrm{l}$ binding buffer containing $5 \mu \mathrm{l}$ of Annexin V-FITC conjugate and $10 \mu \mathrm{l}$ Propidium lodide $(\mathrm{PI})$ solution, and then incubated in the dark at room temperature for $10 \mathrm{~min}$. The apoptotic cells in each group were analyzed using Cell Quest software on a FACScan flow cytometer (Becton Dickinson). A total of 10,000 cells per group were analyzed to quantify the percentage of cells that were Annexin-V- or PI-positive.

\section{Quantitation of atherosclerotic lesions}

Both ligated LCA and non-ligated RCA from mice fed an HFD with or without $0.5 \%(\mathrm{w} / \mathrm{v})$ UDCA were harvested after perfusion with saline and then were immediately embedded in OCT medium and frozen. After fixation with $4 \%$ paraformaldehyde, the sections were stained with Oil-red-O (Bucciarelli et al., 2002). The sizes of atherosclerotic lesions were measured using Image $\mathrm{J}$ software.

\section{Immunofluorescence staining}

Carotid arteries were embedded in OCT medium and then frozen. Sections of $7 \mu \mathrm{m}$ in thickness were prepared. After fixation with $4 \%$ paraformaldehyde, the sections were blocked with $10 \%$ normal serum of goat or donkey in PBS for $1 \mathrm{~h}$. Tissue sections were stained with rabbit anti-XBP-1 (1:50), rabbit anti- 
CHOP (1:50), mouse anti-CD68 (1:50), rabbit anti-VCAM1 (1:50), and rat anti-ICAM1 (1:50), overnight at $4^{\circ} \mathrm{C}$. Samples were incubated for $2 \mathrm{~h}$ with secondary antibody (anti-mouse lgG-R, anti-rabbit lgG-R, or anti-rat lgG-R) in darkness. Nuclei were counterstained with DAPI $(100 \mathrm{ng} / \mathrm{ml}$; Santa Cruz Biotechnology) for $8 \mathrm{~min}$ in the dark. After mounting, tissue slides were observed using an Olympus BX51 (Olympus America).

\section{Statistical analysis}

All data are expressed as means \pm SEMs and represent data from at least three independent experiments. Quantitative variables were tested using the nonparametric Mann-Whitney $U$ test. Differences between groups were considered to be significant at $p<0.05$.

\section{RESULTS}

UDCA reduces OSS-induced ER stress in ECs

Disturbed flow increases activation of XBP-1 expression and splicing in ER stress response in ECs (Zeng et al., 2009), resulting in the progression of atherosclerosis. Furthermore, many studies have reported that UDCA has anti-inflammatory and anti-apoptotic effects in hepatocytes via inhibition of ER stress. So, we explored the possibility that UDCA could inhibit ER stress induced by disturbed flow in ECs. To do this, HUVECs were cultured under LSS or OSS conditions. We confirmed the effects of fluid shear stress on ER stress response in ECs. ER stress levels were measured by quantifying the classical markers of the UPR pathway, including phospho-PERK, phospho-elF2 $\alpha$, XBP-1, p50 ATF6, and CHOP. As shown in Fig $1 \mathrm{~B}$, OSS-cultured ECs showed increases in expression of $\mathrm{XBP}-1$ and $\mathrm{CHOP}$ compared to static conditions. In contrast, expression of XBP-1 was decreased, but there was no change in CHOP expression in ECs under LSS conditions (Fig. 1A). The changes in flow pattern did not affect other ER stress markers such as phospho-PERK, phosphor-eIF2 $\alpha$, and p50 ATF6 (Supplementary Fig. 1). Next, we explored the effects of UDCA on disturbed flow-induced ER stress. UDCA treatment
A
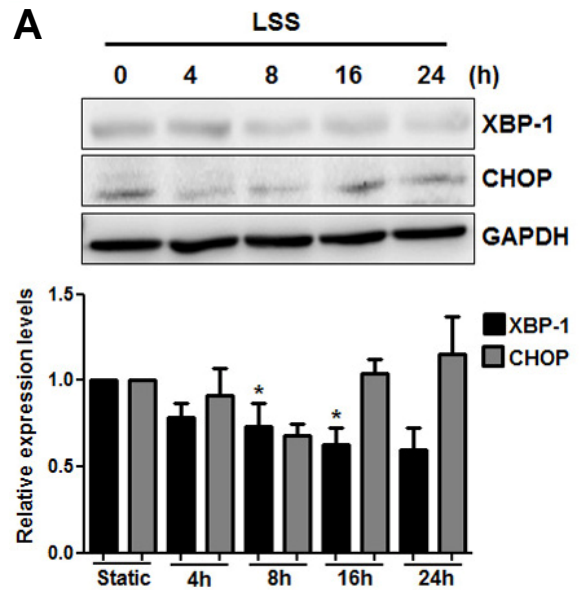

C

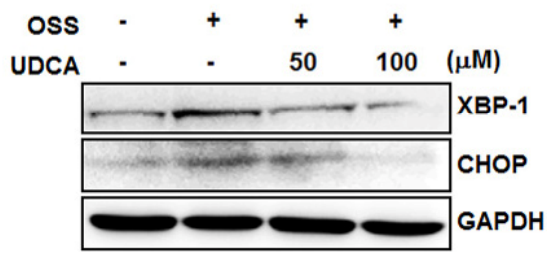

$D$
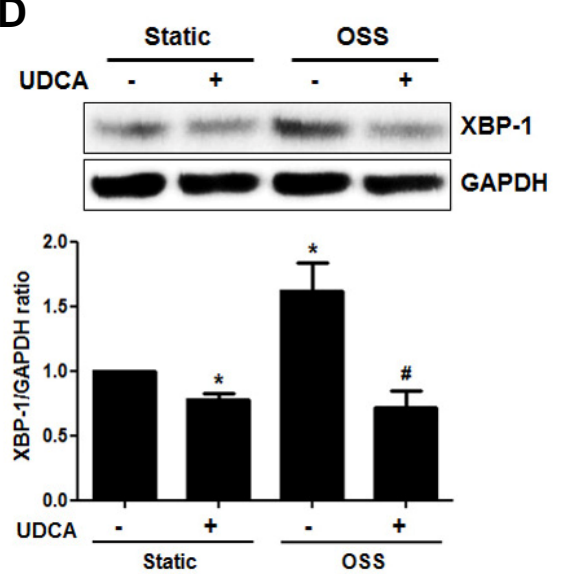

B
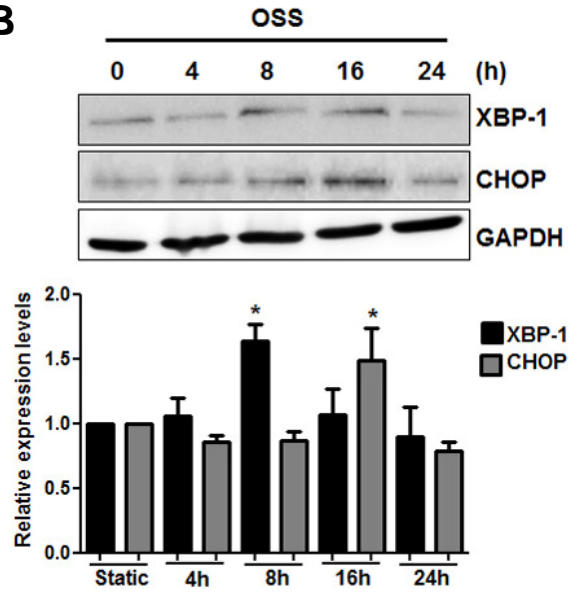

E

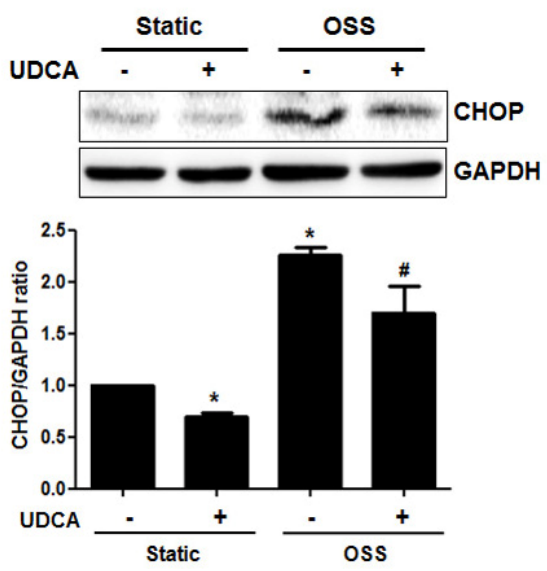

Fig. 1. Effects of UDCA on OSSinduced ER stress in endothelial cells. HUVECs were cultured under static, LSS, or OSS conditions for the indicated time with or without UDCA $(100 \mu \mathrm{M})$. The expression levels of ER stress markers, including XBP-1 and $\mathrm{CHOP}$, were detected by Western blotting. (A, B) Expressions of ER stress markers in endothelial cells under fluid conditions. ${ }^{*} P<0.05$, Static vs. LSS or OSS. Error bars: SEMs. (C-E) Expressions of XBP1and $\mathrm{CHOP}$ in endothelial cells under fluid conditions with or without UDCA. Representative images from at least three experiments are shown. ${ }^{*} P<0.05$, Static vs. Static + UDCA or OSS. \#P $<0.05$, OSS vs. OSS + UDCA. Error bars, SEMs 
reduced disturbed flow-induced expression of XBP-1 and $\mathrm{CHOP}$ in dose-dependent manner, which were most significantly reduced at $100 \mu \mathrm{M}$ of UDCA (Figs. 1C-1E). These results suggest that OSS can induce ER stress and UDCA can inhibit OSS-induced ER stress in ECs.

\section{UDCA inhibits the OSS-induced inflammatory response in ECs}

Recently, it was reported that ER stress-mediated proinflammatory responses are interconnected through various mechanisms, and may also affect the progression of atherosclerosis (Tabas, 2010; Zhang and Kaufman, 2008). Since UDCA inhibited OSS-induced ER stress in ECs, we explored the effects of UDCA on OSS-induced inflammatory responses. It is well known that OSS downregulates anti-inflammatory genes such as eNOS and KLF2, and upregulates proinflammatory genes, such as various adhesion molecules (VCAM-1 and ICAM-1) (Chien, 2007). The levels of protein and mRNA expression in eNOS and KLF2 were significantly reduced in OSS-cultured ECs. However, UDCA successfully recovered decreases in levels of protein and mRNA expression of eNOS and KLF2 in ECs under OSS conditions (Fig. 2A). Additionally, many studies have shown that eNOS phosphorylation is an important factor to regulate eNOS activity. OSS impaired Nitric oxide (NO) production in ECs through downregulation in levels of expression and phosphorylation of eNOS at
$\boldsymbol{A}$

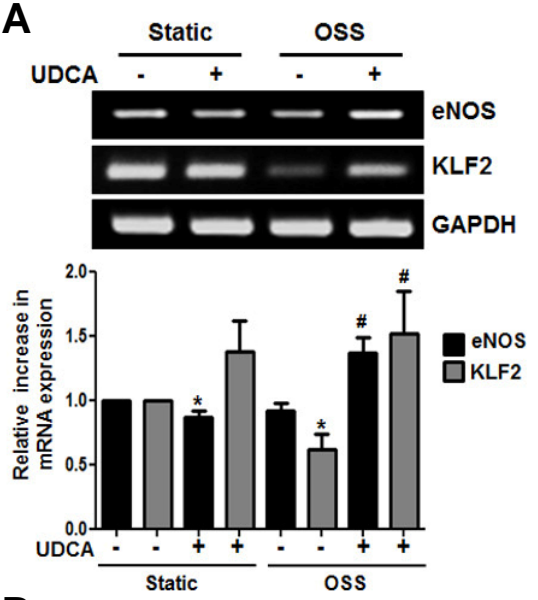

B
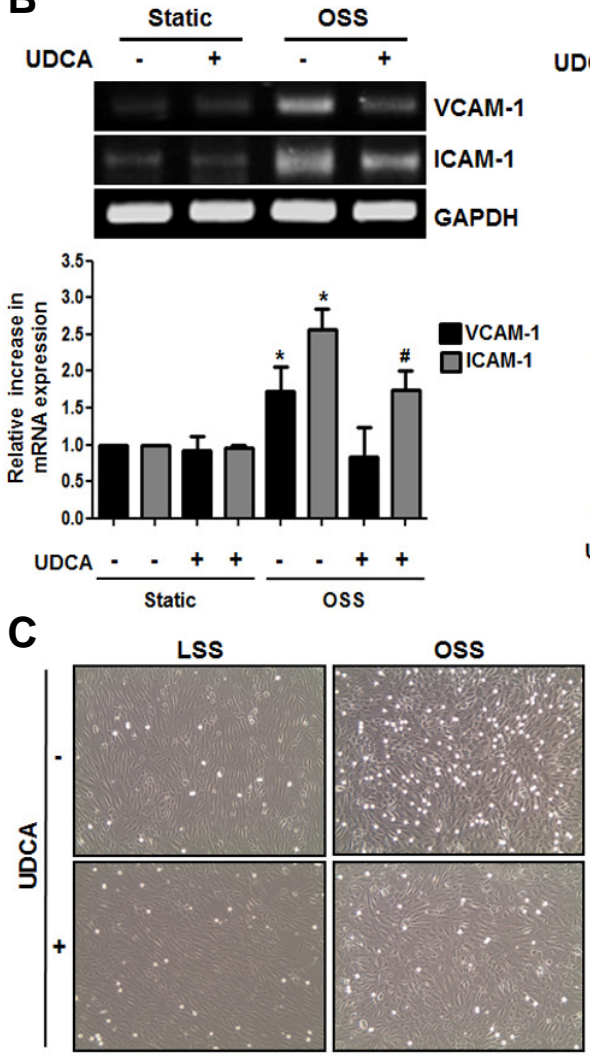
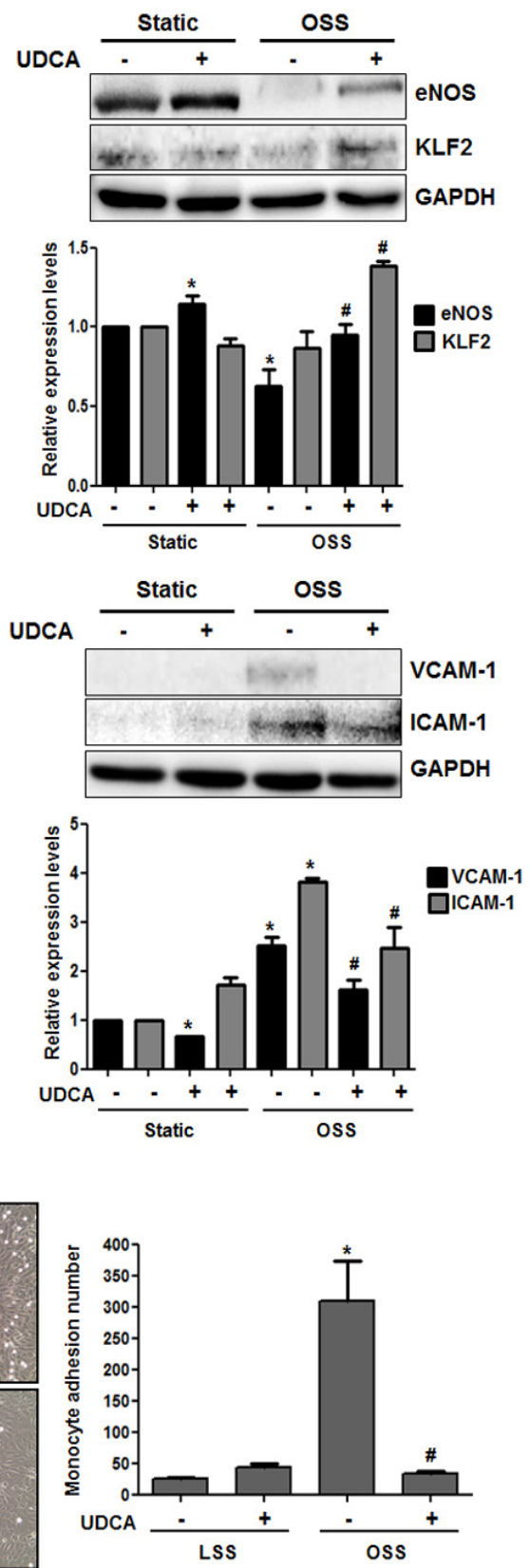

Fig. 2. Effects of UDCA on OSS-induced inflammation in endothelial cells. (A, B) Expressions of anti-inflammatory (eNOS and KLF2) and pro-inflammatory (VCAM-1 and ICAM-1) genes involved in atherosclerosis. HUVECs were cultured under flow or static conditions after pretreatment with UDCA $(100 \mu \mathrm{M})$ for $2 \mathrm{~h}$. The levels of mRNAs encoding eNOS, KLF2, VCAM-1, ICAM-1, and GAPDH (internal control) were measured after 8 hours of flow condition by Reverse transcription (RT)-PCR. The expression levels of eNOS, KLF2, VCAM-1, and ICAM-1 were measured after $24 \mathrm{~h}$ of flow condition by Western blotting. Representative images and quantitative data are shown. ${ }^{*} P<0.05$, Static vs. Static + UDCA or OSS. \#P<0.05, OSS vs. OSS + UDCA. Error bars: SEMs. (C) Monocyte adhesion to endothelial cells. HUVECs were exposed to LSS or OSS conditions with or without UDCA for $24 \mathrm{~h}$. U937 cells were added to endothelial cells and adherent monocytes were counted in five random optical fields in each dish. ${ }^{*} P<0.05$, Static vs. OSS. \#P< 0.05 , OSS vs. OSS + UDCA. Error bars, SEMs. 
Table 1. Metabolic parameters after 2 weeks of study in partially carotid artery ligated ApoE KO mice, with or without UDCA treatment $(n=5$ per group).

\begin{tabular}{lccc}
\hline & HFD & HFD + UDCA & $P$ value \\
\hline Total cholesterol & $1740 \pm 15 \mathrm{mg} / \mathrm{dl}$ & $1261 \pm 147 \mathrm{mg} / \mathrm{dl}$ & 0.0040 \\
Triglyceride & $89 \pm 23 \mathrm{mg} / \mathrm{dl}$ & $91 \pm 39 \mathrm{mg} / \mathrm{dl}$ & 0.5000 \\
HDL & $356 \pm 173 \mathrm{mg} / \mathrm{dl}$ & $77 \pm 14 \mathrm{mg} / \mathrm{dl}$ & 0.0040 \\
LDL & $1300 \pm 228 \mathrm{mg} / \mathrm{dl}$ & $681 \pm 69 \mathrm{mg} / \mathrm{dl}$ & 0.0040
\end{tabular}

Data are expressed as mean \pm SEM. Values were measured in plasma samples. Quantitative variables were compared using the nonparametric Mann-Whitney test. Differences between groups were considered to be significant at $P<0.05$.

$\boldsymbol{A}$

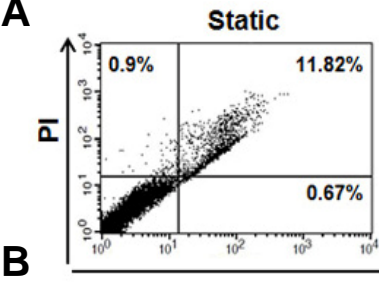

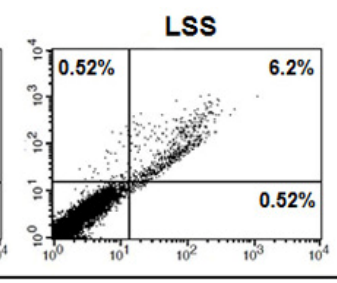
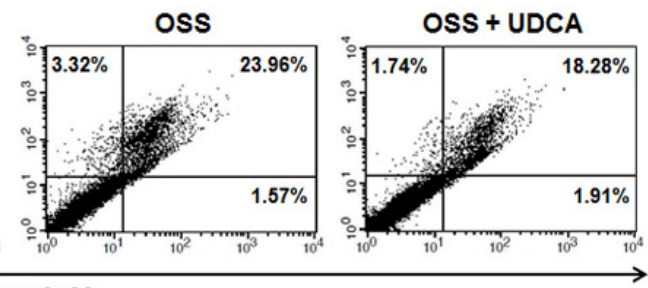

Annexin V

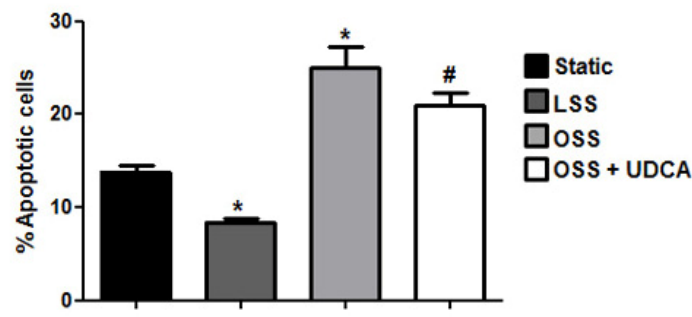

Fig. 3. Effects of UDCA on OSS-induced apoptosis in endothelial cells. HUVECs were cultured under LSS or OSS conditions for $48 \mathrm{~h}$ with or without UDCA $(100 \mu \mathrm{M})$. (A) Apoptosis was assessed by Annexin-V/PI staining using flow cytometry. (B) Quantification of apoptotic cell proportions (\% values). Representative images from at least three experiments and quantitative data are shown. ${ }^{*} P<0.05$, Static vs. Static + UDCA or OSS. \#P< 0.05, OSS vs. OSS + UDCA. Error bars, SEMs.

serine 1177 (Gambillara et al., 2006). So, we next examined the effects of UDCA on phosphorylation of eNOS at serine 1177 in ECs under OSS conditions. As shown in Supplementary Fig. 2, phosphorylation level of eNOS at serine 1177 was reduced under OSS at $30 \mathrm{~min}$. However, UDCA successfully recovered phosphorylation level of eNOS at serine 1177 in ECs which were decreased under OSS conditions. In expression of pro-inflammatory genes, the increases in protein and mRNA levels of VCAM-1 and ICAM-1, triggered by OSS conditions, were significantly inhibited by UDCA (Fig. 2B). To assess the functional relevance of UDCA-mediated suppression of the increase of adhesion molecules by OSS, adhesion of monocytes to ECs was evaluated under LSS and OSS conditions with or without UDCA. As shown in Fig. 2C, the adhesion of monocytes to ECs was markedly increased in OSS-cultured ECs compared to LSS-cultured ECs, but was significantly inhibited by UDCA. Taken together, these results indicate that UDCA exerts anti-inflammatory effects via inhibition of the expression of adhesion molecules induced by OSS.

\section{UDCA inhibits OSS-induced apoptosis in ECs}

Prolonged activation of the ER stress pathway can lead to apoptosis in various cell types and contribute to the progression of atherosclerosis. The overexpression of XBP-1 in ECs triggers endothelial apoptosis via a reduction in expression of en- dothelial junctional adhesion protein VE-cadherin (Zeng et al., 2009). Also, CHOP induces apoptosis by promoting protein synthesis and oxidative stress (Marciniak et al., 2004; Scull and Tabas, 2011; Thorp et al., 2009). Since UDCA inhibited ER stress and reduced expression levels of XBP-1 and $\mathrm{CHOP}$ induced by disturbed flow, we examined the effects of UDCA on disturbed flow-induced apoptosis in ECs. To assess these effects, the ECs cultured under LSS or OSS conditions with or without UDCA were double-labeled with Annexin V-FITC/PI, and analyzed by flow cytometry. In OSS-cultured ECs, the numbers of cells positive for Annexin V-FITC or PI were significantly increased compared to ECs under static or LSS conditions. In contrast, ECs under LSS conditions showed the lowest rate of endothelial apoptosis. However, UDCA treatment reduced OSS-induced apoptosis in ECs (Fig. 3). It is surmised that the reduction in OSS-induced apoptosis in ECs by UDCA was due to suppression of the OSS-induced expression of XBP-1 and CHOP. These results suggest that UDCA may have anti-atherogenic effects via a reduction of OSS-induced apoptosis in ECs.

UDCA reduces atherosclerotic plaque formation induced by disturbed flow in mice

To evaluate the role played by UDCA in disturbed flow-induced atherosclerotic plaque formation, we compared the atheroma- 
tous plaque formation in control and UDCA-treated mice with partially ligated LCA. The size of atheromatous plaque formation was measured by Oil red $O$ staining. In lipid parameters in mice, UDCA-treated HFD group showed lower cholesterol levels in comparison to HFD group except triglyceride (Table 1). The HFD group showed severe atheromatous plaque formation in ligated LCA compared to non-ligated RCA. In contrast, the UDCA-treated HFD group showed significantly less plaque formation in ligated LCA, even in non-ligated RCA (Figs. 4A and $4 B$ ). To explore whether UDCA could inhibit disturbed flowinduced ER stress in ligated LCA of mice, we next observed the expression levels of ER stress markers using immunofluorescence staining. The expression levels of XBP1 and $\mathrm{CHOP}$ were significantly lower in the UDCA-treated HFD group than the HFD group (Figs. 4C and 4E). In addition, subsequent expression levels of adhesion molecules (VCAM-1 and ICAM-1) and infiltration of macrophages (CD68) were detected in ligated LCA. However, the adhesion molecules and infiltration of macrophage were not detectable, or were significantly lower, in the
UDCA-treated HFD group (Figs. 4D and 4E). These results suggest that UDCA reduces atherosclerotic plaque formation via the inhibition of ER stress and inflammatory response induced by disturbed flow.

\section{DISCUSSION}

In the present study, we found that UDCA exerts anti-atherogenic effects in atherosclerosis induced by disturbed flow. UDCA inhibited disturbed flow-induced ER stress, resulting in reductions in XBP-1 and CHOP expression levels. Also, inflammatory responses including increases in adhesion molecules, monocyte adhesion to ECs, and apoptosis of ECs in OSS conditions were reduced by UDCA. In a mouse model of disturbed flow-induced atherosclerosis, UDCA inhibited atheromatous plaque formation and expression of adhesion molecules through the alleviation of ER stress.

Vascular ECs are directly exposed to changes in fluid shear stress, which modulates the function of ECs by activating
$\boldsymbol{A}$

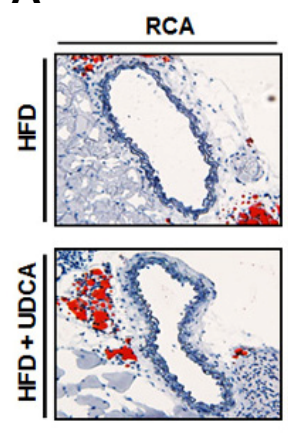

C
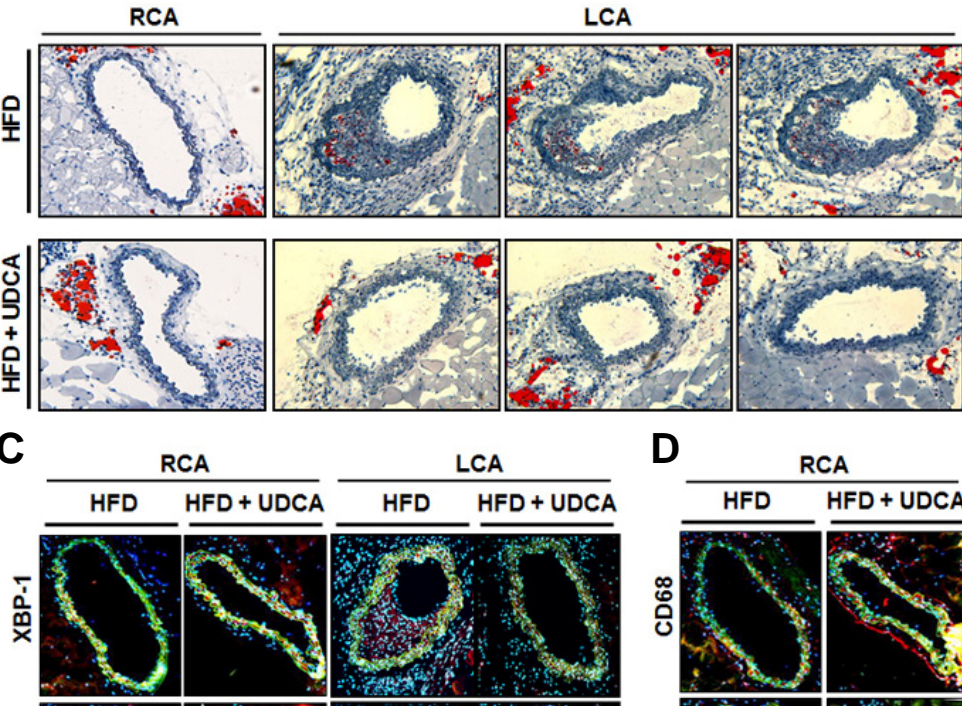

D
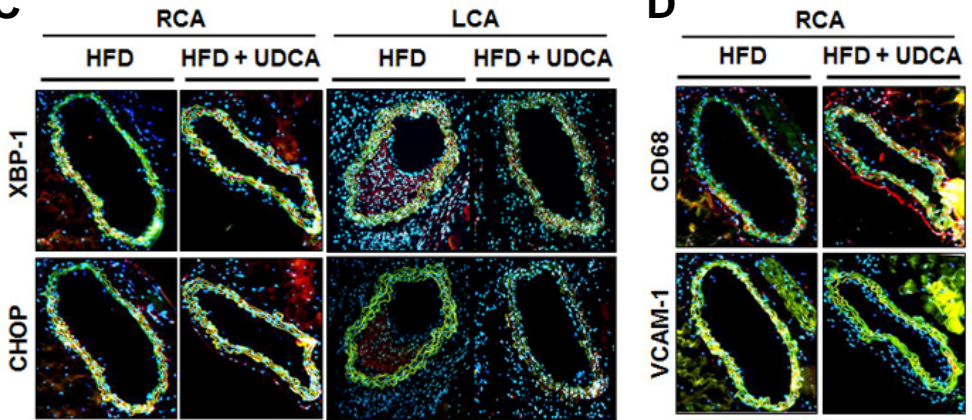

\section{E}

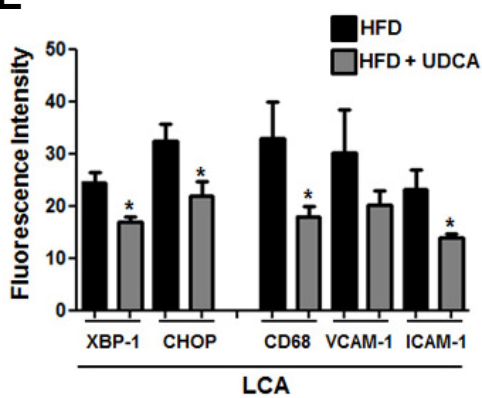

B
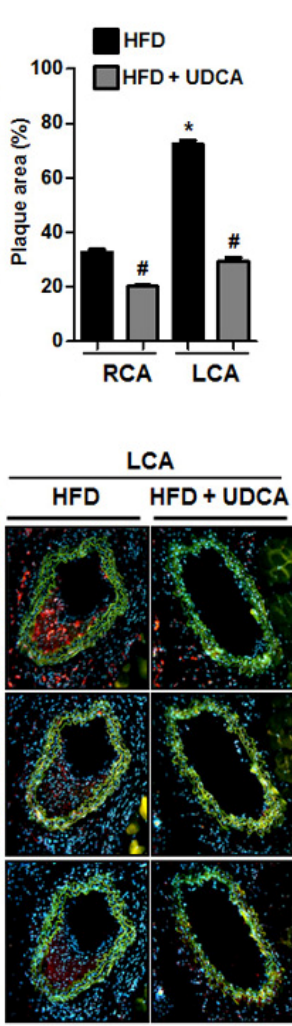

Fig. 4. Effects of UDCA on atherosclerotic plaque formation in mice with partially ligated left carotid artery (LCA). Male ApoE $\mathrm{KO}$ mice were partially ligated in the LCA and fed an HFD with or without $0.5 \%(\mathrm{w} / \mathrm{v})$ UDCA (400$600 \mathrm{mg} / \mathrm{kg} /$ day $/$ mouse) for 2 weeks. (A, B) Quantification of atherosclerotic lesions in carotid arteries. The ligated LCA and nonligated RCA (right carotid artery) of mice were isolated and frozen. Frozen sections from both carotid arteries were stained with Oil red $\mathrm{O}$. To better understand the effect of UDCA in disturbed flow-induced atherosclerotic plaque formation, the plaque areas were compared between the HFD and UDCA-treated HFD groups. Plaque area proportions ( $\%$ values) were quantified using Image $\mathrm{J}$ software. ${ }^{*} P<0.05$, RCA vs. LCA. \#P $<0.05$, HFD vs. HFD + UDCA. Error bars: SEMs. (C, D, and E) Immunofluorescence staining in carotid arteries of mice. ER stress markers (XBP-1 and CHOP) (C) adhesion molecules,

(VCAM-1 and ICAM-1) and a macrophage marker (CD68) (D) are indicated by red signals. The green color is autofluorescence from elastic tissue in the vessel walls. DAPI staining of nuclei is shown in blue and merged images are also presented (magnification, $\times 100$ ). Representative images from at least three experiments are shown. (E) Quantification of red fluorescence intensity in ligated LCA. ${ }^{*}<<$ 0.05 , HFD vs. HFD + UDCA. Error bars, SEMs. 
mechano-sensors, signaling pathways, and expression of genes. Disturbed flow causes endothelial dysfunction and that dysfunction is a critical event in atherosclerosis (Chien, 2007; 2008). There are numerous pieces of evidence that the ER stress pathway is chronically activated in atherosclerotic lesional cells, particularly endothelial cells and macrophages (Tabas, 2010). To this point, inhibition of ER stress induced by disturbed flow has a strategic importance in the prevention and treatment of atherosclerosis. However, there is little evidence regarding any direct correlations between disturbed flow and ER stress in ECs. Recently, disturbed flow was found to directly cause sustained activation of XBP-1 and GRP78 in the ER stress response (Feaver et al., 2008; Zeng et al., 2009). We obtained similar results in that disturbed flow induced activation of the ER stress pathway and elevated the levels of XBP-1 and CHOP. Furthermore, we found that UDCA effectively inhibited ER stress, which was induced by disturbed flow in ECs.

In addition, it was recently shown that the ER stress pathway is directly involved in the induction of inflammation (Zhang and Kaufman, 2008). In our results, expression levels of proinflammatory genes such as adhesion molecules, VCAM-1 and ICAM-1, which were increased in OSS conditions, were significantly suppressed with UDCA treatment. The results of a monocyte adhesion assay in ECs were consistent with a reduction in expression of adhesion molecules by UDCA. Furthermore, expression levels of anti-inflammatory genes such as eNOS and KLF2 were elevated with UDCA treatment in OSS conditions. Moreover, UDCA remarkably recovered the decreased phosphorylation level of eNOS at serine 1177 in ECs under OSS conditions, which is known to involve in regulation of eNOS activity. Therefore, our results imply that UDCA exhibits anti-atherogenic activity by blocking ER stress and the subsequent inflammatory response caused by disturbed flow.

Severe or prolonged ER stress activates the CHOP pathway and it plays crucial roles in the development and progression of cardiovascular and metabolic diseases. The ER stress-CHOP pathway is involved in ER stress-mediated cell damage and it induces apoptosis in various cells (Gotoh et al., 2011). Furthermore, overexpression of XBP-1 in ECs induces apoptosis via decreased expression of VE-cadherin in ECs. Silencing with siRNA specific for CHOP inhibited ER stress-induced apoptosis in ECs and elevated cell viability (Kim et al., 2007). In the present study, we attained similar results, in which UDCA reduced activation of XBP-1 and CHOP in OSS conditions, resulting in a decrease of disturbed flow-induced endothelial apoptosis. Taken together, our results suggest that UDCA reduces disturbed flow-induced endothelial apoptosis via reduced expression of XBP-1 and CHOP, caused by inhibition of ER stress in ECs under disturbed flow.

Consistent with previous studies, we confirmed the presence of increased atheromatous plaque formation, elevated levels of ER stress markers, increased expression of adhesion molecules, and increased infiltration of macrophages in a mouse model of disturbed flow-induced atherosclerosis. In the UDCAtreated HFD group of mice, atheromatous plaque formation was nearly undetectable rather than significantly reduced. The cholesterol profiles was still high levels (over 15-fold in total cholesterol level and 20-fold in LDL) in comparison with them of wild type mice, even though UDCA-treated HFD group have shown lowered cholesterol levels than the HFD group. In this point, remarkable reduction in atheromatous plaque formation in UDCA-treated HFD group is considered that it is just not caused by lowered cholesterol levels. We have found that ER stress markers and the inflammatory response were either undetectable or significantly decreased in the UDCA-treated HFD group. These results can afford explanations a significant reduction in atheromatous plaque formation in UDCA-treated HFD group. In present study, we could not investigate the functional mechanism of UDCA in lipid metabolism. Further studies are required to elucidate the mechanisms of UDCA in lipid metabolism, which may increase our understanding in antiatherogenic activities of UDCA. These results suggest that UDCA might inhibit the development of atherosclerosis caused by disturbed flow by blocking ER stress and its downstream inflammatory response.

In conclusion, we found that UDCA exerts an antiatherogenic activity in atherosclerosis by inhibiting the ER stress and inflammatory response induced by disturbed flow. This study suggests that UDCA may be useful as a therapeutic agent for prevention or treatment of atherosclerosis.

Note: Supplementary information is available on the Molecules and Cells website (www.molcells.org).

\section{ACKNOWLEDGMENTS}

This work was supported by the Bio \& Medical Technology Development Program of the National Research Foundation (NRF) funded by the Korean government (MEST)(NRF-20110019695) and Daewoong Pharmaceutical Co. Ltd.

\section{REFERENCES}

Beuers, U., Boyer, J.L., and Paumgartner, G. (1998). Ursodeoxycholic acid in cholestasis: Potential mechanisms of action and therapeutic applications. Hepatology 28, 1449-1453.

Bucciarelli, L.G., Wendt, T., Qu, W., Lu, Y., Lalla, E., Rong, L.L., Goova, M.T., Moser, B., Kislinger, T., Lee, D.C., et al. (2002). RAGE blockade stabilizes established atherosclerosis in diabetic apolipoprotein E-null mice. Circulation 106, 2827-2835.

Chien, S. (2007). Mechanotransduction and endothelial cell homeostasis: the wisdom of the cell. Am. J. Physiol-Heart C 292, H1209-H1224.

Chien, S. (2008). Effects of disturbed flow on endothelial cells. Ann. Biomed. Eng. 36, 554-562.

Civelek, M., Manduchi, E., Riley, R.J., Stoeckert, C.J., Jr., and Davies, P.F. (2009). Chronic endoplasmic reticulum stress activates unfolded protein response in arterial endothelium in regions of susceptibility to atherosclerosis. Circ. Res. 105, 453461.

Cunningham, K.S., and Gotlieb, A.I. (2005). The role of shear stress in the pathogenesis of atherosclerosis. Lab. Invest. 85, 942-942.

Dai, G.H., Kaazempur-Mofrad, M.R., Natarajan, S., Zhang, Y.Z., Vaughn, S., Blackman, B.R., Kamm, R.D., Garcia-Cardena, G., and Gimbrone, M.A. (2004). Distinct endothelial phenotypes evoked by arterial waveforms derived from atherosclerosissusceptible and -resistant regions of human vasculature. Proc. Natl. Acad. Sci. USA 101, 14871-14876.

Feaver, R.E., Hastings, N.E., Pryor, A., and Blackman, B.R. (2008). GRP78 upregulation by atheroprone shear stress via p38-, alpha 2 beta 1-dependent mechanism in endothelial cells. Arterioscl. Throm. Vas. 28, 1534-1541.

Gambillara, V., Chambaz, C., Montorzi, G., Roy, S., Stergiopulos, N., and Silacci, P. (2006). Plaque-prone hemodynamics impair endothelial function in pig carotid arteries. Am. J. Physiol. Heart Circ. Physiol. 290, H2320-2328.

Gotoh, T., Endo, M., and Oike, Y. (2011). Endoplasmic reticulum stress-related inflammation and cardiovascular diseases. Int. J. Inflamm. 2011, 259462.

Ha, C.H., Wang, W., Jhun, B.S., Wong, C., Hausser, A., Pfizenmaier, K., McKinsey, T.A., Olson, E.N., and Jin, Z.G. (2008). Protein kinase D-dependent phosphorylation and nuclear export of histone deacetylase 5 mediates vascular endothelial growth factor-induced gene expression and angiogenesis. J. Biol. Chem. 283, 14590-14599.

Ha, C.H., Kim, S., Chung, J., An, S.H., and Kwon, K. (2013). 
Extracorporeal shock wave stimulates expression of the angiogenic genes via mechanosensory complex in endothelial cells: Mimetic effect of fluid shear stress in endothelial cells. Int. J. Cardiol. 168, 4168-4177.

Heo, K.S., Fujiwara, K., and Abe, J. (2014). Shear stress and atherosclerosis. Mol. Cells 37, 435-440.

Hotamisligil, G.S. (2010). Endoplasmic reticulum stress and atherosclerosis. Nat. Med. 16, 396-399.

Kim, K.M., Pae, H.O., Zheng, M., Park, R., Kim, Y.M., and Chung, H.T. (2007). Carbon monoxide induces heme oxygenase-1 via activation of protein kinase $\mathrm{R}$-like endoplasmic reticulum kinase and inhibits endothelial cell apoptosis triggered by endoplasmic reticulum stress. Circ. Res. 101, 919-927.

Kim, S.Y., Kwon, Y.W., Jung, I.L., Sung, J.H., and Park, S.G. (2011). Tauroursodeoxycholate (TUDCA) inhibits neointimal hyperplasia by suppression of ERK via PKCalpha-mediated MKP-1 induction. Cardiovasc. Res. 92, 307-316.

Lin, J.H., Li, H., Yasumura, D., Cohen, H.R., Zhang, C., Panning, B. Shokat, K.M., LaVail, M.M., and Walter, P. (2007). IRE1 signaling affects cell fate during the unfolded protein response. Science 318, 944-949.

Lindor, K. (2007). Ursodeoxycholic acid for the treatment of primary biliary cirrhosis. New Engl. J. Med. 357, 1524-1529.

Ma, J., lida, H., Jo, T., Takano, H., Oonuma, H., Morita, T., Toyo-Oka, T., Omata, M., Nagai, R., Okuda, Y., et al. (2004). Ursodeoxycholic acid inhibits endothelin-1 production in human vascular endothelial cells. Eur. J. Pharmacol. 505, 67-74.

Marciniak, S.J., Yun, C.Y., Oyadomari, S., Novoa, I., Zhang, Y.H., Jungreis, R., Nagata, K., Harding, H.P., and Ron, D. (2004). $\mathrm{CHOP}$ induces death by promoting protein synthesis and oxidation in the stressed endoplasmic reticulum. Gene Dev. 18, 3066-3077.

Nam, D., Ni, C.W., Rezvan, A., Suo, J., Budzyn, K., Llanos, A., Harrison, D., Giddens, D., and Jo, H. (2009). Partial carotid ligation is a model of acutely induced disturbed flow, leading to rapid endothelial dysfunction and atherosclerosis. Am. J. Physiol. Heart Circ. Physiol. 297, H1535-H1543.

Passerini, A.G., Polacek, D.C., Shi, C.Z., Francesco, N.M., Manduchi, E., Grant, G.R., Pritchard, W.F., Powell, S., Chang,
G.Y., Stoeckert, C.J., et al. (2004). Coexisting proinflammatory and antioxidative endothelial transcription profiles in a disturbed flow region of the adult porcine aorta. Proc. Natl. Acad. Sci. USA $101,2482-2487$.

Scull, C.M., and Tabas, I. (2011). Mechanisms of ER StressInduced Apoptosis in Atherosclerosis. Arterioscl. Throm. Vas. 31 2792-2797.

Tabas, I. (2009). Macrophage apoptosis in atherosclerosis: consequences on plaque progression and the role of endoplasmic reticulum stress. Antioxid. Redox Signal. 11, 23332339.

Tabas, I. (2010). The role of endoplasmic reticulum stress in the progression of atherosclerosis. Circ. Res. 107, 839-850.

Thorp, E., Li, G., Seimon, T.A., Kuriakose, G., Ron, D., and Tabas, I. (2009). Reduced apoptosis and plaque necrosis in advanced atherosclerotic lesions of $\mathrm{Apoe}^{-/-}$and $\mathrm{Ldll}^{-/-}$mice lacking CHOP. Cell Metab. 9, 474-481.

Timmins, J.M., Ozcan, L., Seimon, T.A., Li, G., Malagelada, C., Backs, J., Backs, T., Bassel-Duby, R., Olson, E.N., Anderson M.E., et al. (2009). Calcium/calmodulin-dependent protein kinase II links ER stress with Fas and mitochondrial apoptosis pathways. J. Clin. Invest. 119, 2925-2941.

Zeng, L., Zampetaki, A., Margariti, A., Pepe, A.E., Alam, S., Martin, D., Xiao, Q., Wang, W., Jin, Z.G., Cockerill, G., et al. (2009) Sustained activation of XBP1 splicing leads to endothelial apoptosis and atherosclerosis development in response to disturbed flow. Proc. Natl. Acad. Sci. USA 106, 8326-8331.

Zhang, K.Z., and Kaufman, R.J. (2008). From endoplasmicreticulum stress to the inflammatory response. Nature 454, 455462.

Zhou, J., Lhotak, S., Hilditch, B.A., and Austin, R.C. (2005). Activation of the unfolded protein response occurs at all stages of atherosclerotic lesion development in apolipoprotein Edeficient mice. Circulation 111, 1814-1821.

Zinszner, H., Kuroda, M., Wang, X.Z., Batchvarova, N., Lightfoot, R.T., Remotti, H., Stevens, J.L., and Ron, D. (1998). CHOP is implicated in programmed cell death in response to impaired function of the endoplasmic reticulum. Gene Dev. 12, 982-995. 